\title{
Desperdício de medicamentos no ambulatório em Portugal
}

Zilda Mendes, ${ }^{*}$ Sofia Crisóstomo, ${ }^{* *}$ Francisco Batel Marques, ${ }^{* * *}$ Ana Paula Martins, ${ }^{* * * *}$ Vitor Rodrigues, ${ }^{* * * * *}$ Carlos Fontes Ribeiro*****

\section{RESUMO}

Objectivos: Caracterizar a dimensão do desperdício de medicamentos, no ambulatório, em Portugal, em quantidade e valor, devido a: 1) inadequação da dimensão da embalagem ao tempo de tratamento necessário e 2) não adesão do doente à terapêutica. Tipo de Estudo: Prospectivo Longitudinal.

Local: Estudo Nacional com recolha de informação, entre Setembro de 2005 e Setembro de 2006, nas farmácias e via telefone. População e Métodos: Inquérito através de entrevista na farmácia, com recolha de informação sobre o indivíduo e a terapêutica instituída. Seguimento telefónico, no final da duração do tratamento (situações agudas) ou no final previsto da(s) embalagem(ns) adquirida(s) (terapêutica para doenças crónicas). Eram elegíveis os doentes com 18 anos ou mais, com uma prescrição de pelo menos um medicamento sujeito a receita médica, em formas orais sólidas, comparticipado pelo SNS e destinado ao início de um tratamento (excluindo a medicação para tomar em situação de recurso). O desperdício foi avaliado através do número de unidades farmacêuticas, percentagem de doses diárias definidas (DDD) e encargos desperdiçados. Foi implementado um algoritmo de simulação, que permitiu encontrar, qual(is) a(s) dimensão(ões) de embalagens que deveriam estar disponíveis no mercado português por forma a minimizar o desperdício.

Resultados: Amostra composta por 1.601 doentes, os quais adquiriram 2.098 medicamentos. Da amostra em estudo, 68,6\% eram doentes do sexo feminino e tinham idade média de 50 anos. O desperdício global foi de $21,7 \%$ da quantidade prescrita em DDD. Cerca de metade $(9,7 \%)$ deste desperdício global foi atribuído à dimensão da embalagem dispensada e o restante $(10,2 \%)$ à não adesão à terapêutica. O custo global associado ao desperdício atingiu, em média, 4,44 € por medicamento.

Conclusões: A dimensão do desperdício encontrado pode constituir uma base de fundamentação sólida para estudos futuros que permitam optimizar a informação disponível aos decisores nesta área. A simulação efectuada demonstrou ser possível diminuir grande parte desse desperdício, se estivessem disponíveis outros tamanhos de embalagens. O desperdício associado à não adesão à terapêutica deve ser abordado na prática clínica com vista a optimizar os resultados em saúde dos doentes.

Palavras-chave: Dimensionamento das Embalagens de Medicamentos; Desperdício de Medicamentos; Adesão à Terapêutica; Farmacoepidemiologia.

\section{INTRODUÇÃO}

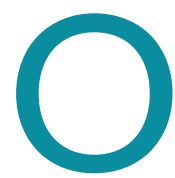

uso racional dos medicamentos pressupõe que os doentes recebam os medicamentos apropriados para as suas necessidades terapêuticas, em doses e quantidades ajustadas aos tempos de tratamento e ao mais baixo custo possível. ${ }^{1}$ Assim, a dimensão das embalagens constitui uma componente da terapêutica racional, cuja inadequação, aliada à não adesão à terapêutica, foi identificada como factor gerador de ineficiência na alocação dos recursos de saúde. ${ }^{2-9}$ Além do desperdício de recursos escassos, os medicamentos não utilizados podem ainda resultar num risco acrescido de diminuição da efectividade, ${ }^{10-13}$ intoxicação acidental ${ }^{14,15}$ ou contaminação ambiental. ${ }^{16-18}$
*(Estatística - Mestre em Probabilidades e Estatística) Directora Adjunta do Centro de Estudos e Avaliação em Saúde

**(Farmacêutica - Licenciada em Ciências Farmacêuticas, Mestranda em Desenvolvimento, Diversidades Locais e Desafios Mundiais) Consultora

***(Farmacêutico - Doutorado) Professor da Faculdade de Farmácia da Universidade de Coimbra

****(Farmacêutica - Doutorada) Professora da Faculdade de Farmácia da Universidade de Lisboa
*****(Médico - Doutorado) Professor da Faculdade de Medicina da Universidade de Coimbra

*****(Médico - Doutorado) Professor da Faculdade de Medicina da Universidade de Coimbra

Entidades promotoras do estudo:

Associação Nacional das Farmácias: Centro de Estudos e Avaliação em Saúde; Instituto da Qualidade em Saúde; Administração Regional de Saúde do Centro 
Várias metodologias têm sido utilizadas para avaliar o desperdício associado à utilização de medicamentos, quer através da análise dos medicamentos com prazo de validade expirado e/ou já não utilizados (devolvidos à farmácia ${ }^{3,5,19}$ ou armazenados no domicílio dos doentes $\left.^{2,7-9}\right)$, quer através dos registos individuais de dispensa de medicamentos. ${ }^{4,6}$ No entanto, nenhum dos estudos mencionados distinguiu entre desperdício devido à inadequação da quantidade de medicamento prescrita e desperdício associado à não adesão à terapêutica por parte dos utilizadores.

Em Portugal, um estudo piloto realizado, em $2002 / 3,{ }^{20}$ na região Centro, evidenciou que a prevalência do desperdício por inadequação das embalagens aos tempos de tratamento propostos e por não adesão dos doentes à terapêutica era de 30,1\% e 46,7\%, respectivamente. De salientar ainda que em 2004 foram objecto de valorização energética cerca de 390 toneladas de medicamentos, recolhidos pelas Farmácias Portuguesas, no âmbito do projecto VALORMED. ${ }^{21}$

\section{OBJECTIVO}

O presente estudo teve como objectivos caracterizar e quantificar o desperdício de medicamentos e respectivos custos associados, em Portugal, no âmbito do Serviço Nacional de Saúde (SNS), quer devido à inadequação da dimensão das embalagens dos medicamentos, quer devido à não adesão dos doentes à terapêutica.

\section{METODOLOGIA}

Modelo de estudo: Foi realizado um estudo prospectivo longitudinal, com recolha de informação inicial através de entrevista pessoal efectuada na farmácia (mediante questionário estruturado) e posterior seguimento dos doentes através de entrevista telefónica.

Dimensão da amostra: Com os valores de desperdício encontrados num estudo piloto, ${ }^{20}$ foi estimada uma dimensão de amostra global de 2.582 doentes. Esta dimensão garantiria, para um nível de confiança de 95\%, obter estimativas da prevalência do desperdício a nível nacional com um erro inferior a $2 \%$ e a nível regional com um erro máximo de $10 \%$.

Selecção da amostra/recolha de informação: Foram convidadas a participar neste estudo todas as farmácias de Portugal Continental associadas da Associação Nacional das Farmácias (ANF), tendo sido prestada forma- ção a todos os farmacêuticos que aceitaram participar no estudo. A distribuição regional das farmácias participantes no estudo foi comparada com a distribuição regional do universo, para verificar que todas as regiões estavam representadas na amostra em proporção semelhante à do universo.

Foram considerados elegíveis para o estudo os doentes com 18 anos ou mais, aos quais foi prescrito e dispensado pelo menos um medicamento (sem exclusão de classes terapêuticas) na forma oral sólida, sujeito a receita médica, comparticipado pelo SNS e destinado ao início de um tratamento (primeira vez que o doente toma o medicamento ou, não sendo a primeira vez, não tome o medicamento há mais de 3 meses). Os medicamentos prescritos sem duração de terapêutica definida quantitativamente foram excluídos da análise (ex. utilização em situação de recurso).

Foi analisada a hora de recrutamento dos doentes para o estudo, de forma a verificar que tinham sido recrutados doentes aleatoriamente durante todos os períodos do dia.

No caso dos doentes darem o seu consentimento informado para serem contactados posteriormente, a(s) embalagem(ns) dispensada(s) foram marcadas com etiquetas autocolantes e, através de contacto telefónico, foi recolhida informação sobre a quantidade de unidades farmacêuticas (comprimidos, cápsulas, saquetas, etc.) remanescentes. $\mathrm{O}$ original do consentimento informado foi enviado para o CEFAR juntamente com o restante material de estudo e, quer o doente, quer o farmacêutico ficaram com uma cópia. O seguimento telefónico foi feito imediatamente após o final do tratamento (situações agudas), ou no final da(s) embalagem(ns) dispensada(s) (terapêutica nas doenças crónicas), por uma equipa de entrevistadores do IQS com formação previa sobre os objectivos do estudo e técnicas de entrevista telefónica (no máximo foram efectuadas 3 tentativas de contacto).

Definição de conceitos: O desperdício de medicamentos foi definido como a quantidade de medicamento prescrito e dispensado que não foi utilizado para o tratamento instituído no momento da recolha de informação inicial.

Dimensão inadequada da embalagem - diferença entre o número de unidades farmacêuticas prescritas (tamanho da embalagem x número de embalagens) e 
o número de unidades farmacêuticas necessárias para finalizar o tratamento prescrito na receita em análise (número de unidades/dia x número de dias de tratamento). Este diferencial designou-se por número de UF desperdiçadas $\left(\mathrm{n}_{\mathrm{UF}}\right)$. Este número foi igualmente transformado em número de doses diárias definidas (DDD), dose definida pela OMS para estudos de utilização de medicamentos.

Não adesão à terapeûtica - avaliada em função das sobras, que foram reportadas pelo doente na entrevista telefónica, efectuada no final do tempo previsto para o tratamento instituído na receita em análise (data de aquisição da embalagem + número de dias necessários ao tratamento prescrito na receita +1 ).

Análise estatística: Para avaliação de diferenças foi utilizado o teste de Kruskal-Walis ou o teste de Qui-Quadrado para tabelas de contingência (dependendo da natureza das variáveis independentes e dependentes em análise). A análise dos custos foi efectuada com base no preço de venda a público dos medicamentos e parte comparticipada pelo SNS, uma vez que foi solicitada cópia (anónima) do talão de aquisição do(s) medicamento(s). Foi também ajustado um modelo de regressão logística para avaliação dos determinantes do desperdício por não adesão á terapêutica. Por último, para algumas substâncias foi realizado um estudo de simulação, para determinar que dimensão(ões) de embalagem(ns) minimizariam o desperdício encontrado.

\section{RESULTADOS}

\section{Caracterização da amostra}

Participaram no estudo 10,0\% das farmácias de Portugal Continental associadas da ANF $(n=261)$, distribuídas por todas as regiões de Portugal Continental e em proporção semelhante à do Universo de farmácias $(p=0,216)$. Não se verificaram igualmente diferenças significativas da proporção de farmácias participantes, porTipologia de Áreas Urbanas ${ }^{22}(\mathrm{p}=0,059)$, face ao Universo das farmácias.

Entre 21 de Setembro de 2005 e 10 de Abril de 2006, foram convidados a participar no estudo 1.926 doentes que cumpriam os critérios de inclusão, dos quais, 176 $(9,1 \%)$ recusaram participar no inquérito realizado na farmácia (maioritariamente referindo falta de tempo para a entrevista). Dos restantes doentes, 149 foram excluídos da análise por inconsistências no preenchimen- to do questionário. Dos 1.601 doentes incluídos no estudo, $1.473(92,0 \%)$ deram o seu consentimento informado para serem seguidos telefonicamente. A principal razão para a recusa de participação na fase prospectiva do estudo foi o facto de os doentes não gostarem de ser contactados por esse meio $(n=63)$. Não se registaram diferenças significativas por sexo $(\mathrm{p}=0,121)$ e por idade ( $\mathrm{p}=0,554)$, quando comparados os participantes e os não participantes no seguimento telefónico.

Dos 1.601 doentes incluídos na análise inicial, a maioria $(68,4 \%)$ era do sexo feminino e com idade média de 50 anos (desvio padrão=17). Relativamente à escolaridade, mais de metade $(55,3 \%)$ dos doentes tinha só o ensino básico ou sabia apenas ler e escrever.

\section{Caracterização da medicação}

Os 1.601 doentes incluídos no estudo adquiriram 2.098 medicamentos, num total de 2.275 embalagens dispensadas.

Segundo a Classificação Anatómica, Terapêutica e Química (ATC) ${ }^{23}$, as classes mais representadas na amostra foram: J01 - Antibacterianos para uso sistémico ( $\mathrm{n}=464 ; 22,1 \%)$, M01 - Anti-inflamatórios e antireumáticos (n=286; 13,6\%), R06 - Anti-histamínicos para uso sistémico ( $\mathrm{n}=142 ; 6,8 \%), \mathrm{C} 10$ - Fármacos hipolipidemiantes ( $n=119 ; 5,7 \%)$ e A02- Anti-ácidos e modificadores da secreção gástrica $(\mathrm{n}=116 ; 5,5 \%)$. Salienta-se que a elevada frequência de dispensa de antibacterianos para uso sistémico e vacinas é característica do período em que decorreu o recrutamento (entre Setembro de 2005 e Abril de 2006).

Os médicos de família foram responsáveis pela prescrição de $71,3 \%$ dos medicamentos.

\section{Caracterização do desperdício}

O número médio de UF desperdiçadas foi de 7,7 unidades por medicamento, correspondendo a 6,7 DDD. Do total de DDD dispensadas, foram desperdiçadas $21,7 \%$ - Quadro I.

Cerca de metade $(9,7 \%)$ foi desperdiçada devido à inadequação da dimensão da embalagem face ao esquema terapêutico prescrito e o restante devido à não adesão à terapêutica $(10,2 \%)$. No que respeita aos custos, foi desperdiçado, em média, o correspondente a $4,44 €$ por medicamento. Deste desperdício, $60,4 \%$ foi suportado pelo SNS. Não foram encontradas diferen- 
ças estatisticamente significativas entre as regiões de saúde ( $p>0,05)$, para qualquer dos indicadores avaliados (Figura 1).
Para 10 classes terapêuticas mais representadas na amostra em estudo, os Quadros II e III apresentam os Índices de Desperdício (em DDD) e os respectivos custos associados. No que respeita à análise em número e $\%$ de DDD, a classe terapêutica com maior desperdício foi a M01, no entanto, no que diz respeito a custos a classe A02 foi a que apresentou maior custo desperdiçado.

No que respeita à inadequação da dimensão da embalagem prescrita/dispensada, o maior desperdício foi encontrado para a nimesulida (12,2 UF; $41,3 \%$ ), a desloratadina (5,1 UF; 25,7\%) e a associação de amoxicilina e ácido clavulânico $(2,7$ UF; 11,6\%).

Relativamente à nimesulida (200 mg), o número de UF mais frequentemente prescritas foi de $6 \mathrm{UF}, 10 \mathrm{UF}$ e $20 \mathrm{UF}$ e as embalagens disponíveis no mercado eram de 10, 14, 30 e 60 UF - Figura 2. O estudo de simulação revelou que o desperdício poderia ser minimizado para $0,5 \mathrm{UF}$, em média, se coexistissem no mercado embalagens de 6 UF e 10 UF e/ou múltiplos destas.

No caso da desloratadina (5mg), apenas estava disponível e comparticipada a embalagem de 20 UF - Figura 3. Neste caso, o desperdício poderia ser reduzido para 0,7 UF, em média, se existissem outras dimensões de embalagem múltiplos de 5 UF. As embalagens mais dispensadas de amoxicilina e ácido clavulânico (875/125 mg), foram as de 24 UF $(30,7 \%)$ e 16 UF $(28,8 \%)$ - Figura 4. O desperdício poderia ser reduzido para $0,1 \mathrm{UF}$, em média, se a embalagem de 16 UF fosse disponibilizada e se fossem prescritas embalagens mais próximas do número de unidades necessárias para o tratamento.

Relativamente à não adesão à terapêutica prescrita, não foram 


\begin{tabular}{|c|c|c|c|}
\hline \multirow[b]{2}{*}{ Classe ATC } & \multirow[b]{2}{*}{$\mathrm{n}^{*}$} & \multicolumn{2}{|c|}{ DDD desperdiçadas/medicamento } \\
\hline & & $\begin{array}{c}\mathrm{n}^{* *} \\
{[\mathrm{IC} 95 \%]}\end{array}$ & $\begin{array}{c}\% * * * \\
{[\text { IC } 95 \%]}\end{array}$ \\
\hline A02 - Anti-ácidos e modificadores da secreção gástrica & 91 & $\begin{array}{c}8,9 \\
{[6,4-11,5]}\end{array}$ & $\begin{array}{c}22,7 \\
{[16,6-28,9]}\end{array}$ \\
\hline C05 - Vasoprotectores & 45 & $\begin{array}{c}4,6 \\
{[2,75-6,45]}\end{array}$ & $\begin{array}{c}15,3 \\
{[9,1-21,5]}\end{array}$ \\
\hline C09 - Fármacos que actuam no sist. renina-angiotensina & 78 & $\begin{array}{c}7,0 \\
{[3,0-11,0]}\end{array}$ & $\begin{array}{c}11,7 \\
{[6,4-17,1]}\end{array}$ \\
\hline C10 - Fármacos anti-hiperlipidémicos & 88 & $\begin{array}{c}6,5 \\
{[3,7-9,4]}\end{array}$ & $\begin{array}{c}10,4 \\
{[6,2-14,5]}\end{array}$ \\
\hline J01 - Antibióticos para uso sistémico & 383 & $\begin{array}{c}1,9 \\
{[1,5-2,3]}\end{array}$ & $\begin{array}{c}11,3 \\
{[9,2-13,3]}\end{array}$ \\
\hline J07 - Vacinas & 59 & $\begin{array}{c}4,3 \\
{[2,4-6,2]}\end{array}$ & $\begin{array}{c}14,4 \\
{[8,2-20,5]}\end{array}$ \\
\hline M01 - Anti-inflamatórios e anti-reumatismais & 234 & $\begin{array}{c}9,0 \\
{[7,6-10,4]}\end{array}$ & $\begin{array}{c}38,8 \\
{[34,8-42,9]}\end{array}$ \\
\hline N05 - Psicolépticos & 66 & $\begin{array}{c}4,5 \\
{[2,5-6,4]}\end{array}$ & $\begin{array}{c}24,0 \\
{[16,5-31,5]}\end{array}$ \\
\hline N06 - Psicoanalépticos & 86 & $\begin{array}{c}5,6 \\
{[3,0-8,2]}\end{array}$ & $\begin{array}{c}14,5 \\
{[8,7-20,3]}\end{array}$ \\
\hline R06 - Anti-histamínicos para uso sistémico & 122 & $\begin{array}{c}7,9 \\
{[6,7-9,1]}\end{array}$ & $\begin{array}{c}38,9 \\
{[33,2-44,6]}\end{array}$ \\
\hline Total & 1708 & $\begin{array}{c}6,7 \\
{[5,5-7,8]}\end{array}$ & $\begin{array}{c}21,7 \\
{[20,3-23,2]}\end{array}$ \\
\hline
\end{tabular}

*número de medicamentos avaliáveis; ${ }^{* *}$ número médio de DDD desperdiçadas por medicamentos; ${ }^{* * *}$ percentagem média de DDD desperdiçadas por medicamento.

QUADRO III. Encargos (€) desperdiçados, por classe ATC
\begin{tabular}{l|c|c} 
& \\
& & Encargos desperdiçados/medicamento \\
\hline Classe ATC & $n^{*}$ & $€^{* *}[$ IC95\%] \\
\hline A02 - Anti-ácidos e modificadores da secreção gástrica & 90 & $11,04[7,89-14,20]$ \\
\hline C05 - Vasoprotectores & 45 & $3,29[1,97-4,61]$ \\
\hline C09 - Fármacos que actuam no sist. renina-angiotensina & 75 & $4,18[2,08-6,28]$ \\
\hline C10 - Fármacos anti-hiperlipidémicos & 86 & $4,80[2,78-6,82]$ \\
\hline J01 - Antibióticos para uso sistémico & 371 & $2,82[2,29-3,35]$ \\
\hline J07 - Vacinas & 57 & $2,78[1,56-4,00]$ \\
\hline M01 - Anti-inflamatórios e anti-reumatismais & 229 & $6,60[5,51-7,70]$ \\
\hline N05 - Psicolépticos & 65 & $2,06[1,05-3,07]$ \\
\hline N06 - Psicoanalépticos & 86 & $4,30[2,64-5,96]$ \\
\hline R06 - Anti-histamínicos para uso sistémico & 118 & $3,12[2,64-3,60]$ \\
\hline Total*** & 1667 & $4,44[4,02-4,86]$
\end{tabular}

*número de medicamentos avaliáveis; **média de encargos desperdiçados por medicamento; ***PVP não disponível para 41 medicamentos. 


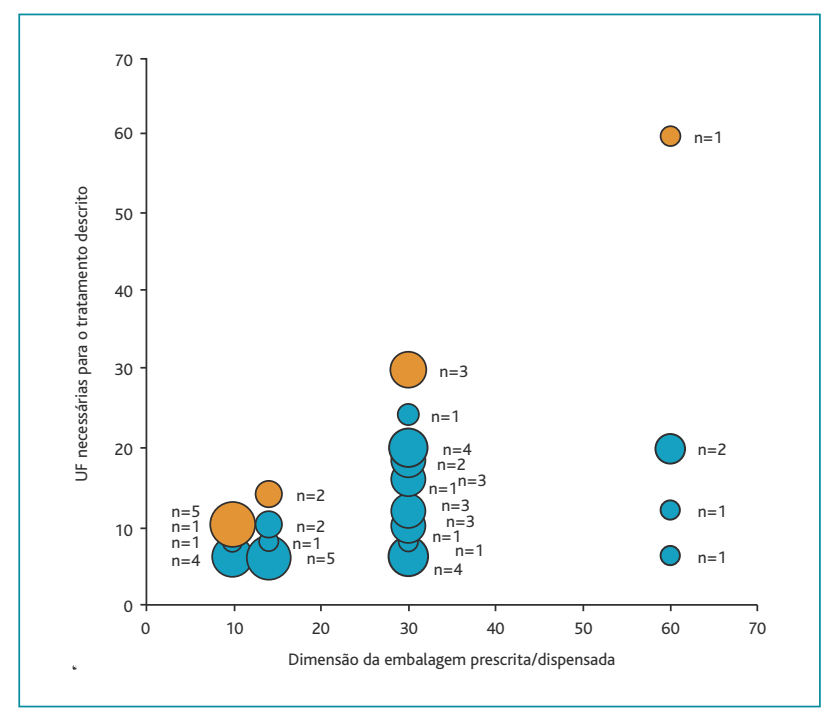

Figura 2. Quantidade de UF necessárias para o tratamento prescrito com Nimesulida (200 mg) e respectiva dimensão de embalagem prescrita (dimensão das bolhas proporcional ao número de doentes em cada situação).

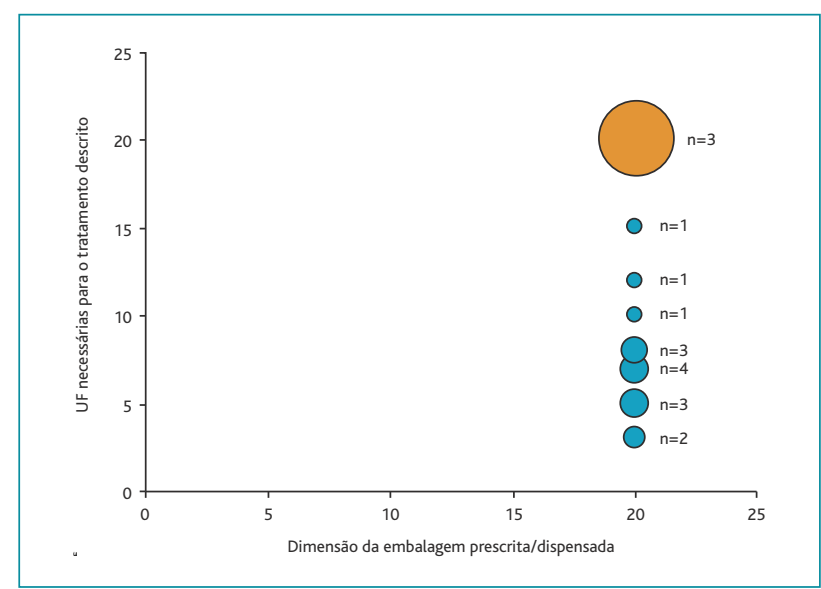

Figura 3. Quantidade de UF necessárias para o tratamento prescrito com Desloratadina e respectiva dimensão de embalagem prescrita (dimensão das bolhas proporcional ao número de doentes em cada situação).

encontradas diferenças estatisticamente significativas por sexo $(\mathrm{p}=0,3587)$, grupo etário $(\mathrm{p}=0,5134)$ e nível de escolaridade $(p=0,2692)$, mas foram encontradas diferenças significativas em relação ao número de medicamentos que o doente estava a adquirir $(\mathrm{OR}=1,41$ [IC95\%:1,02-1,95]; $\mathrm{p}=0,0363$; um versus mais do que um).

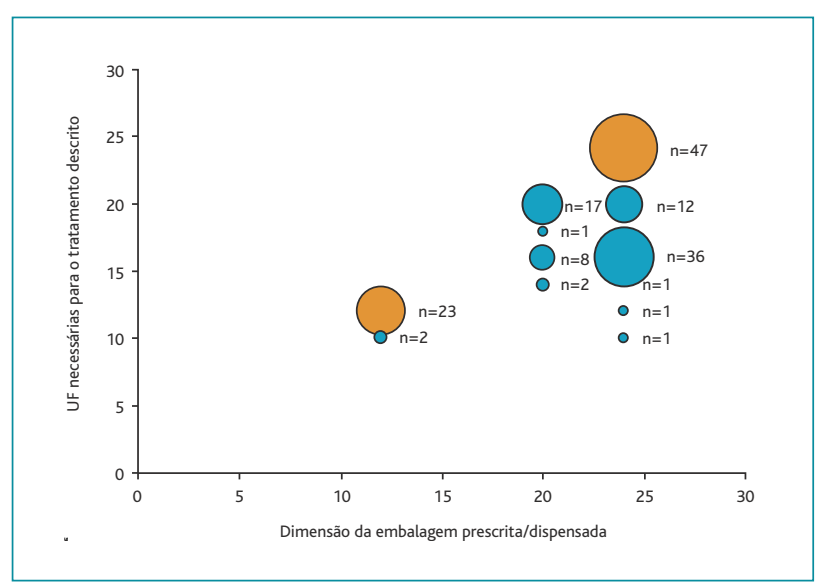

Figura 4. Quantidade de UF necessárias para o tratamento prescrito com Amoxicilina e Ácido Clavulânico (875/125 mg) e respectiva dimensão de embalagem prescrita (dimensão das bolhas proporcional ao número de doentes em cada situação)

O desperdício médio por medicamento, associado à não adesão à terapêutica foi maior, no caso da simvastatina $(8,6 \% ; 14 €)$, da associação de diosmina e hesperidina $(8,7 \% ; 14 €)$ e do omeprazol $(4,5 \% ; 9 €)$.

\section{DISCUSSÃO}

Do desperdício observado, cerca de metade deveu-se à inadequação da dimensão da(s) embalagem(ns) ao tratamento instituído e o restante à não adesão dos doentes à terapêutica prescrita. Estudos realizados na Arábia Saudita e Irão, que analisaram os medicamentos guardados no local de residência, identificaram, valores de desperdício acima dos $25 \%$, correspondendo a mais de $19 \%$ do custo total da medicação. ${ }^{2,9} \mathrm{Na}$ Alemanha, estudos evidenciaram que as embalagens devolvidas às farmácias continham, em média, $65 \%$ do conteúdo original, e $24 \%$ continham mesmo a totalidade do respectivo conteúdo. ${ }^{3}$ Cerca de $60 \%$ do desperdício foi suportado pelo SNS. Valor ligeiramente superior (75\%), foi também encontrado num estudo realizado em Barcelona. ${ }^{19}$ Foi encontrado desperdício em várias classes ATC, tal como em outros estudos analisados ${ }^{2,3,5,9}$, mas a sua hierarquização dependeu do indicador em análise. Relativamente à dimensão das embalagens existentes, o estudo de simulação efectuado permitiu verificar que a adequação daqueles às reais necessidades da prescrição pode minimizar bastante o desperdício. Esta matéria tem sido objecto de análise e já fo- 
ram efectuadas duas revisões da legislação. A primeira através da Portaria n. ${ }^{\circ}$ 1278/2001, de 14 de Novembro e a segunda pela Portaria n. ${ }^{\circ} 1471 / 2004$, de 21 de Dezembro. Durante e na sequência deste estudo, houve redimensionamento, por exemplo, de algumas embalagens de antibacterianos pelo que a realização deste tipo de ensaios pode contribuir para a monitorização das medidas de racionalização do uso do medicamento. Este estudo foi realizado através de questionários delineados para o efeito, com base nas variáveis de interesse operacionalizadas de acordo com a tipologia dos dados e respectiva construção dos indicadores de resultados. Em virtude da informação que constitui estes indicadores ser, essencialmente, de natureza quantitativa e semi-quantitativas, não se considerou determinante proceder a uma validade de conteúdo dos instrumentos. Foi contudo adoptado um procedimento de verificação obrigatória dos medicamentos inscritos no questionário através do talão de venda anonimizado, uma vez que a duração da terapêutica só poderia ter sido validada com recurso ao médico prescritor.

Relativamente ao desperdício associado à não adesão à terapêutica, apenas foi encontrada associação com o número de medicamentos que o doente está a tomar (um versus mais do que um), facto que reforça a ideia de que a polimedicação pode influenciar a adesão á terapêutica.

Não foram encontradas diferenças significativas na distribuição regional do desperdício. No entanto, há que ter em conta que, os intervalos de confiança das estimativas de desperdício por região são bastante alargados, por não se ter atingido a dimensão de amostra desejável (apesar do apelo ao reforço no recrutamento de doentes em Janeiro de 2006).

$\mathrm{O}$ facto de só se terem considerado medicamentos em formas orais sólidas e utilizadores incidentes, limita a inferência destes resultados para a população em geral. No entanto, as formas orais sólidas constituem a maioria dos medicamentos prescritos e dispensados nas farmácias e, em alguns países, a maioria (64\%) dos medicamentos devolvidos às farmácias. ${ }^{5}$

O conhecimento prévio por parte dos doentes de que iriam ser contactados telefonicamente poderá ter influenciado o indicador de desperdício associado à adesão à terapêutica, e neste caso as estimativas apresentadas nesta componente do estudo poderão estar estimadas por defeito. Por outro lado, no caso de terapêuticas crónicas, a eventual quantidade em sobra, poderia ser consumida na continuação do tratamento e, nesta situação, as estimativas apresentadas para este tipo de terapêuticas poderão estar sobre-estimadas. No entanto, como a entrevista telefónica foi realizada no final do tempo previsto para a duração da(s) embalagem(ns) prescrita(s) e dispensada(s), qualquer desperdício encontrado decorre da definição de desperdício utilizada neste estudo, que inclui as situações em que o medicamento não foi consumido no tempo previsto. Esta definição foi adaptada do conceito de uso racional do medicamento utilizado pela OMS ${ }^{1}$.

Neste estudo não foram avaliados alguns aspectos, que parecem estar relacionados com o desperdício e que justificariam uma análise mais detalhada para melhor medição e explicitação do fenómeno. Factores como a não adesão induzida por suspensão da terapêutica (devido a efeitos adversos de medicamentos ou à sua não efectividade) deveriam ser analisados em estudos futuros pelo impacto que a suspensão da terapêutica pode assumir na quantificação do desperdício relacionado com o uso não racional do medicamento.

Podemos concluir que:

- O desperdício global identificado neste estudo foi de $21,7 \%$ da quantidade prescrita em unidades farmacêuticas. Cerca de metade $(9,7 \%)$ deveu-se à inadequação da dimensão da(s) embalagem(ns) ao tratamento instituído, e a outra metade $(10,2 \%)$ à não adesão dos doentes à terapêutica. Em termos económicos, o desperdício global atingiu, em média, $4,44 €$ por medicamento. O co-financiamento do SNS representou $60,4 \%$ do total de encargos desperdiçados. Foi encontrado desperdício em todas as classes ATC, quer por inadequação da dimensão da embalagem, quer por não adesão à terapêutica. A simulação efectuada permitiu verificar que um reajuste das dimensões de embalagens de medicamentos, pode minimizar grande parte do desperdício identificado.

Os resultados encontrados de não adesão à terapêutica merecem reflexão devendo ser abordados na prática clínica com vista a optimizar os resultados em saúde dos doentes. 
1. The rational use of drugs: report of the conference of experts, Nairobi, 25-29 November 1985. Geneva:World Health Organization, 1987.

2. Abou-Auda HS. An economic assessment of the extent of medication use and wastage among families in Saudi Arabia and Arabian Gulf countries. Clin Ther 2003 Apr; 25 (4): 1276-92.

3. Bronder E, Klimpel A. Unused drugs returned to the pharmacy - new data. Int J Clin Pharmacol Ther 2001 Nov; 39 (11): 480-3.

4. Domino ME, Olinick J, Sleath B, Leinwand S, Byrns PJ, Carey T. Restricting patients' medication supply to one month: saving or wasting money?. Am J Health Syst Pharm 2004 Jul; 61 (13): 1375-9.

5. Garey KW, Johle ML, Behrman K, Neuhauser MM. Economic consequences of unused medications in Houston, Texas. Ann Pharmacother 2004 Jul-Aug; 38 (7-8): 1165-8.

6. Kahan NR, Chinitz DP, Kahan E. Longer than recommended empiric antibiotic treatment of urinary tract infection in women: an avoidable waste of money. J Clin Pharm Ther 2004 Feb; 29 (1): 59-63.

7. Morgan TM. The economic impact of wasted prescription medication in an outpatient population of older adults. J Fam Pract 2001 Sep;50 (9): 779-81.

8. Wasserfallen JB, Bourgeois R, Büla C, Yersin B, Buclin T. Composition and cost of drugs stored at home by elderly patients. Ann Pharmacother 2003 May; 37 (5): $731-7$.

9. Zargarzadeh $\mathrm{AH}$, Tavakoli $\mathrm{N}$, Hassanzadeth A. A survey on the extent of medication storage and wastage in urban Iranian households. Clin Ther 2005 Jun; 27 (6): 970-8.

10. Burman WJ, Cohn DL, Rietmeijer CA, Judson FN, Sbarbaro JA, Reves RR. Noncompliance with directly observed therapy for tuberculosis: epidemiology and effect on the outcome of treatment. Chest 1997 May; 111 (5): 1168-73.

11. Lesaffre E, Kocmanová D, Lemos PA, Disco CM, Serruys PW. A retrospective analysis of the effect of noncompliance on time to first major adverse cardiac event in LIPS. Clin Ther 2003 Sep; 25 (9): 2431-47.

12. Miura T, Kojima R, Mizutani M, Shiga Y, Takatsu F, Suzuki Y. Effect of digoxin noncompliance on hospitalization and mortality in patients with heart failure in long-term therapy: a prospective cohort study. Eur J Clin Pharmacol 2001 Apr; 57 (1): 77-83.

13. Waterman AD, Miligan PE, Bayer L, Banet GA, Gatchel SK, Gage BF. Effect of warfarin nonadherence on control of the International Norma- lized Ratio. Am J Health Syst Pharm 2004 Jun 15; 61 (12): 1258-64.

14. Centers for Disease Control and Prevention. Nonfatal, unintentional medication exposures among young children - United States, 2001-2003. MMWR Morb Mortal Wkly Rep 2006 Jan 13; 55 (1): 1-5.

15. Dean BS, Krenzelok EP. Poisoning in the elderly: an increasing problem for health care providers. J Toxicol Clin Toxicol 1987; 25 (5): 411-8.

16. Boxall $A B$. The environmental side effects of medication. EMBO Rep 2004 Dec; 5 (12): 1110-6.

17. Rooklidge SJ. Environmental antimicrobial contamination from terraccumulation and diffuse pollution pathways. Science Total Environ 2004 Jun $5 ; 325$ (1-3): 1-13.

18. Zuccato E, Castiglioni S, Fanelli R, Reitano G, Bagnati R, Chiabrando C, et al. Pharmaceuticals in the environment in Italy: causes, occurrence, effects and control. Environ Sci Pollut Res Int 2006 Jan; 13 (1): 15-21.

19. Coma A, Modamio P, Lastra CF, Bouvy ML, Mariño EL. Returned medicines in community pharmacies of Barcelona, Spain. Pharm World Sci 2008 Jun; 30 (3): 272-7.

20. Mendes Z, Martins AP, Ferreira G, Batel Marques F, Rodrigues V. Desperdício na Utilização de Medicamentos na Região Centro. Lisboa: CEFAR; 2004.

21. Carapeto J. O Sistema Integrado de Gestão de Resíduos de Embalagens e Medicamentos - SIGREM: Objectivos e Resultados no período 2000-2004. Seminário "Gestão de Resíduos Na Fileira do Medicamento". Lisboa. 22-6-2006.

22. Instituto Nacional de Estatística, Direcção Geral do Ordenamento do Território e Desenvolvimento Urbano. Indicadores Urbanos do Continente 1999. Lisboa: Instituto Nacional de Estatística; 1999.

23. World Health Organization Collaborating Centre for Drug Statistics Methodology: ATC classification index with including daily defined doses. Janeiro 2006.

Os autores declararam não possuir conflitos de interesses

\section{ENDEREÇO PARA CORRESPONDÊNCIA}

Zilda Mendes

Centro de Estudos e Avaliação em Saúde

Rua Marechal Saldanha, n. ${ }^{0} 1$

1249-069 Lisboa, Portugal

Recebido em 03/02/2009

Aceite para publicação em 21/11/2009 


\section{ABSTRACT}

\section{DRUGS WASTAGE IN AMBULATORY CARE IN PORTUGAL}

Objective: To characterize the amount of medicines wasted in ambulatory care, Portugal, measured in quantity and value, due to: 1) inadequacy of medicines package sizes to the therapeutic regimen and 2) non-compliance to the treatment prescribed. Study Design: Prospective study.

Local: National study carried out between September 2005 and September 2006.

Population and Methods: First a questionnaire was administered in the pharmacy, in order to identify the patient and the therapeutic regimen prescribed.A second questionnaire was developed for telephone interview and applied after the predicted length of treatment (acute conditions) or at the end of the package size (therapeutics for chronic diseases). Inclusion criteria were: patients aged 18 or over, with at least one prescription from the Portuguese National Health Service and oral therapies for a first time use (excluding emergency medication). Wastage was measured in number of pharmaceutical units, percentage of daily defined doses (DDD) and costs. A simulation study was performed to determine which package sizes would minimize the wastage due to the inadequacy of package sizes.

Results: A sample of 1.601 patients which purchase 2.098 medicines. The mean age was 50 years old and $68.6 \%$ of patients were females. The global wastage was identified in $21.7 \%$ of the quantity prescribed in DDD. About one half $(9,7 \%)$ was due to the inadequacy of the pack sizes, and the remaining $(10,2 \%)$ due to non-compliance to the treatment prescribed. The average wastage cost per medicine dispensed was $4.44 €$

Conclusions: Medicines wastage found in this national study could be a good support for upcoming studies that can lead to optimize information for decision makers in this area. The simulation study showed that this could be reduced if other package sizes where available. Wastage due to non-compliance should be targeted in clinical practice in order to optimize patients' health related outcomes.

Keywords: Package Size; Medicines Wastage; Compliance to Therapy; Pharmacoepidemiology.

\section{MANUEL BORRALHO}

Manuel Borralho nasceu em Amareleja (Moura) em 1943. Licenciou-se em medicina e exerce a profissão em Mourão desde 1981. É um auto-didacta que gosta de passar os seus tempos livres a desenhar e pintar. Expôs os seus trabalhos por duas vezes: a primeira numa exposição colectiva na Herdade do Esporão em Maio de 2001; a segunda - em que apresentou um conjunto de aguarelas com o título "Aguarelas de Alqueva» - teve lugar na Galeria Municipal de Mourão e decorreu em Fevereiro de 2008.

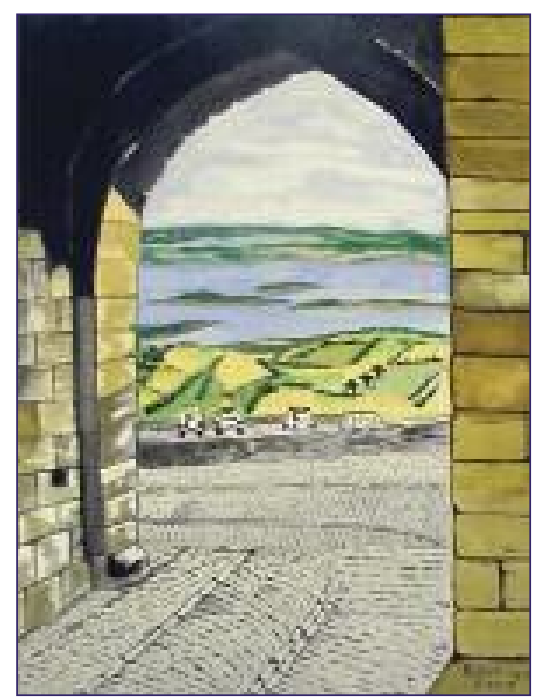

Pintura do Alqueva vista do Castelo de Monsaraz Manuel Borralho 